\title{
Parental Attitudes and the Development of the Primary Transitional Object
}

\author{
Fred Busch, Ph.D.* \\ University of Michigan Medical Center \\ and \\ Judith McKnight, M.S.W. \\ University of Michigan Medical Center
}

\begin{abstract}
Research on the primary transitional object has ignored the subtle interactions that occur between parents and children in the development and use of the primary transitional object. While conscious expectations of whether the child will develop a primary transitional object do not seem to be an important factor, unconscious motivations seem to determine if parents serve as either facilitators or disturbers of the child's relationship to the primary transitional object. The ways in which parental attitudes may affect the development of the primary transitional object, and the consequences of this, are described.
\end{abstract}

*Dr. Busch is Assistant Professor of Psychology, Children's Psychiatric Hospital, University of Michigan Medical Center, Ann Arbor, Michigan 48104. Miss McKnight is Clinical Social Worker, Children's Psychiatric Hospital, University of Michigan Medical Center. This research was conducted under the auspices of the Child Psychoanalytic Study Program (Director, Humberto Nagera, M.D.) of Children's Psychiatric Hospital, University of Michigan Medical Center. The authors express gratitude to Dr. Guillermo Pezzarossi for his help in interviewing the mothers of the children in the study. 
In conducting studies of the primary transitional object* we were surprised at the complexity and variety of parental attitudes toward the primary transitional object. The basis for our surprise was that in all previous studies of the primary transitional object there has been an absence of or a one-sided elaboration of parental attitudes toward the transitional object. Winnicott [2,3] and Gaddini [4] both stressed the role of the "good enough mother" in the development of the transitional object, but do not discuss the mother's attitude toward the object and the role that this may play in the child's relationship to it. Stevenson [5] stresses that in her sample of mothers the stance toward the child's transitional object was primarily positive. As an example of this positive attitude toward the transitional object Stevenson [5: p. 204] quotes one mother as saying, "When will he give it up? I don't know, but I do know that I shall never insist. I feel when he no longer needs it, he will do so of his own accord. It is too deeply loved and has helped him through too many trials to be too easily discarded." At the opposite end of the continuum Sperling [6] describes pathological unconscious factors in a mother's attitude that may lead the child to have an inordinate attachment to an object, and she comes to the conclusion that the child's attachment to a transitional object is an unhealthy development.

In summary, there has been little discussion of parental attitudes toward the transitional object and the role this may play in the development of the transitional object. Where discussed the view presented seems to be one-sided, and does not match our own experience where a complex set of attitudes toward the primary transitional object is expressed by parents. Thus, a natural step for us was to investigate parental attitudes toward the primary transitional object as a factor in its development.

\footnotetext{
*In our investigative efforts [1] we have found it necessary to distinguish between two types of objects, each having properties of Winnicott's $[2,3]$ "transitional object," but which seem to serve differing developmental purposes. Those objects to which the child develops an attachment in the first year of life we have labeled the "primary transitional object." This is to be distinguished from those object attachments that occur at around age two which we have called the "secondary transitional object." The prototypical primary transitional object is a soft, malleable object that develops around six months of age from a set of experiences around going to sleep.
} 


\section{Method of Study}

A study of 40 children from 23 different families was conducted to investigate the qualities of the child's relationship to the primary transitional object. Twenty-seven of the 40 children showed evidence of having an attachment to a primary transitional object. The great majority of families were those who had children enrolled in the normal toddler and nursery school programs of the Child Psychoanalytic Study Program. The population is an educationally skewed one, with a large percentage of families having at least one member associated with a large university as either an educator, researcher, or advanced graduate student. Data from families of staff members within the Child Psychoanalytic Study Program were also included. These staff members all had children that were either currently involved in or barely beyond the use of the primary transitional object, who initially illuminated a particular point of interest in the study.

Extensive interviews with mothers of the children were conducted. These interviews were generally semistructured. Although there were certain specific areas regarding the development of the primary transitional object that we attempted to cover, interviewers were free to follow unique and interesting trends that were presented by parents. The mothers seemed to feel comfortable with this method of inquiry, and there was often a great deal of spontaneous material brought forth by them.

Since the study of the parental attitudes toward the primary transitional object was not the main focus of our initial investigation, analysis of the data was retrospective. We were helped in this retrospective analysis by detailed records of the interviews. It was also the impression of all the interviewers that the mothers were usually open in expressing their view of the child's attachment to the primary transitional object, thus allowing for a variety of clinical inferences to be made.

\section{Results}

\section{Expectations}

In our sample we found that parents had very different expectations of whether or not their child would develop an attachment to a primary transitional object. These expectations 
ranged from mothers who knew nothing about primary transitional objects and thus did not anticipate their child becoming attached to one, to those mothers who very much wanted their child to have a primary transitional object. Many gradations and variations along this continuum were found. However, it seems that conscious parental expectations of whether or not the child will develop an attachment to a primary transitional object has little to do with the development of the primary transitional object.

A large percentage of parents in our study did not in any way anticipate that their child would become attached to a primary transitional object, but yet such an attachment did occur. On the other hand, a number of parents intentionally supplied an object for the explicit purpose that the child would have a primary transitional object, and subsequently the child did develop an attachment to that particular object. Still another group of mothers attempted the same thing, but the object was subsequently rejected by the child. At times the object rejected was replaced by another. Thus, Mrs. H. hoped her son would use a stuffed dog as his primary transitional object, and tied it into his crib in hopes that this would eventuate. It was reported that this "never turned him on," but subsequently the child developed an attachment to a blanket. Thus it appears that while parents may act as facilitators in providing a constant object for the child, this is no guarantee that the child will choose this object as his primary transitional object.

While conscious parental expectations seem not to play a role in the infant's attachment to a primary transitional object, we certainly do not rule out unconscious factors. The relationship of the parental unconscious to the infant's primary transitional object was seen most clearly in the discovery of the attachment by the parents. A common occurrence in our sample was for a mother faced with a fussy baby to suddenly seize upon the idea of getting the infant's blanket. When given the blanket, the infant would immediately become calm. In reconstructing this event, it would appear that the parents had "known" of the infant's attachment to an object for some time, but not on a conscious level. For example, special care would be taken to bring the infant's blanket along on a vacation even though there was no practical reason for this. This type of incident appeared frequently in those families where there was no expectation of the child's attachment to a primary transitional object. It appeared in these families that conscious awareness of the infant's attachment to the primary transitional object was preceded by a preconscious perception. 
Here we see how unconscious factors can affect whether parents serve a facilitating or disruptive role in the infant's attachment to a primary transitional object. Given that in all infants who develop an attachment to the primary transitional object that the object has been a part of the crib since birth [1], the preconscious perception of the infant's beginning attachment to an inanimate object could be acted upon positively or negatively without conscious censorship. We would hypothesize that it is at this point of preconscious perception of the infant's attachment to an object that parents will choose, on the basis of unconscious factors, to allow the child to have the primary transitional object or to take it away for a variety of "rational" reasons. In these cases the parents would not consciously know of the attachment to a primary transitional object nor of their disruption of the process.

In summary, it would appear that while parents may not be able to choose whether their child will become attached to a primary transitional object, there is a minimum requirement of allowing the child to have a potential primary transitional object consistently available. It is in this latter area where unconscious factors might play a role in the determination of whether attachment occurs.

\section{Attitudes}

That parents have an extraordinary sensitivity to the infant's reliance on the primary transitional object can be seen in a number of ways. When asked what they believe the primary transitional object means to the infant, parents responded with such things as: "a buffer to the world"; "a memory of infancy"; "a nursing substitute"; and "helps the child in transition to strange places." These are all common meanings ascribed to the primary transitional object in the psychoanalytic literature $[2,3,7,8]$. Thus it seems evident that many parents were closely attuned to the meaning of the primary transitional object for their child.

Parental empathy with the child's use of the primary transitional object was also seen in their attempts to supply the object for the child whenever it was necessary. For example, great care is usually taken by the parents to pack the primary transitional object when the family is going to be away from home for any length of time. The custom of cutting a child's blanket into two or 
more indistinguishable parts seems to be primarily a response to the child's anxiety over having the entire blanket washed, and thus not available to him when he needs it. An evening trip to the laundromat to soothe a screaming child whose primary transitional object was not dry enough to take to bed was one of the many sacrifices by parents in respect for the child's need for the primary transitional object.

In contrast to the above, many mothers show a good deal of anxiety and concern over their child's use of the primary transitional object. Between the ages of two and three, it is not uncommon for parents to start resenting the child's reliance on the primary transitional object and consider it a "nuisance." Underlying this attitude seem to be two important feelings. The first of these is that the child's continued reliance on the primary transitional object represents a deficiency in the mother-child relationship. Implied in this is the feeling that, as parents, they have done something wrong to the child. For example, one mother felt that her child's great need for the primary transitional object was due to her "cutting down too quickly on his bottles when he was an infant." Yet there is no evidence to indicate that the development of the primary transitional object is related to early weaning, and all signs seemed to indicate that this mother handled weaning quite sensibly.

A second important feeling that many parents experience is the worry that their child will become "overdependent" on the primary transitional object. This often leads to restrictions being placed on the child's use of the primary transitional object. For example, various parents restricted the use of the primary transitional object to times when the child was in the house, or naptime and bedtime, or when the child was sick. The major reason given for restrictions placed on the primary transitional object was the fear that its use would spread, and the child would no longer be interested in the usual pursuits of a toddler. As one mother stated, "It would prevent the child from playing with other things." However, what emerged in those families where no overt restrictions were placed on the primary transitional object was that the child would ultimately regulate its use on his own. That is, at times of stress the child may have used the primary transitional object a great deal, but at other times it would be used only at naptime and bedtime.

As one might expect, then, parental attitudes toward the primary transitional object are characterized by a mixture of 
sensitivity and anxiety. While recognizing its great importance to the child, many parents view it conflictually and end up resenting the primary transitional object.

\section{Discussion}

Our data essentially corroborate Winnicott's [2, 3] finding that the primary transitional object is "created" by the infant. That is, conscious parental expectations do not seem to play a role in whether the infant develops an attachment to the primary transitional object. Parents do not give the child the primary transitional object. Rather, the infant discovers and creates the primary transitional object and its uses for himself. The major parental role in attachment seems to be at an unconscious level as a facilitator. That is, the parent may or may not allow the child the freedom to engage in attachment to a primary transitional object. Thus with many parents who did not anticipate their child's attachment to a primary transitional object, their perception of the infant's tie to the primary transitional object came before conscious awareness. This attachment was not interfered with by them, and the infant's attachment was allowed to develop.

What is striking is that even when parents are sensitive to the child's use of a primary transitional object, it is fertile ground for the arousal of conflictual feelings. Guilt over inadequate parenting and conflicts over gratification of dependency needs are two of the primary difficulties that were prominent in our sample of parents. These feelings appeared to be the main motivations for the restrictions on the child's use of the primary transitional object. The desire for restrictions on the child's use of the primary transitional object seemed based mainly on parental needs in that those children who had no restrictions placed on the use of the primary transitional object engaged in self-regulation of its use.

Given our agreement with Tolpin [8] that the primary transitional object as a soother aids greatly in the separationindividuation process, we would see restrictions of the primary transitional object as a disruptive factor in a critical developmental process. It should be pointed out that the types of restrictions we are discussing are those that appeared arbitrary-for example, those times when limits were placed on the use of the primary transitional object that seemed based on parental anxieties. There 
are other times when the primary transitional object may serve other than adaptive purposes, and the sensitive mother may well attempt to interfere with this process. Thus, there may be occasions when the primary transitional object is used defensively as did one three-year-old in our sample who incorporated it within a compulsive ritual whenever he was faced with stress. It is at these times that a parent may well restrict the use of the primary transitional object, while helping the child to find more effective means of dealing with stress. However, the example cited is not typical of the restrictions seen in our sample. As indicated earlier, the restrictions placed on use of the primary transitional object in our sample seemed primarily a function of parental anxieties rather than the child's needs. Furthermore, the child using the primary transitional object as part of a compulsive defense is no longer using it as part of a normal, developmental process leading to structure and self-soothing.

The nature of the child's reaction to arbitrary restrictions placed on the use of the primary transitional object will very likely depend on the type of restriction, along with the developmental stage of the child. For example, we would expect a different reaction in the one-year-old who is suddenly allowed to use his blanket only at bedtime and not at other times of stress, from that of the three-year-old who is no longer allowed to take his primary transitional object outside. It is likely that the three-year-old would handle the stressful situation more easily, in part because of the greater ability for self-soothing based on use of the primary transitional object and the internalization of its functions [8]. Within our own sample, arbitrary restrictions were usually instituted between the ages of two and three. When these restrictions were placed on the child's use of the primary transitional object, the predominant initial response from the child was that of rage. This seemed indicative of the fact that an important process had been interfered with. We would suggest that what has been interfered with has been an important adjunct in narcissistic development, the primary transitional object, and its role in separation-individuation.

\section{References}

1. Busch F, Nagera H, McKnight J, et al : Primary transitional objects. $J$ Amer Acad Child Psychiat, 12: 193-214, 1973. 
2. Winnicott DW: Transitional objects and transitional phenomena. Int $J$ Psychoanal 34:89-97, 1953.

3. Winnicott DW: Preface to The first treasured possession, by $O$ Stevenson. Psychoanal Study of the Child 9:199-201, 1954.

4. Gaddini $R$ : Transitional objects and the process of individuation: A study in three different social groups. $J$ Amer Acad Child Psychiat 9:347-64, 1970 .

5. Stevenson O: The first treasured possession. Psychoanal Study of the Child 9:199-217, 1954.

6. Sperling M: Fetishism in children. Psychoanal $Q$ 32:374-92, 1963.

7. Greenacre P: The fetish and the transitional object. Psychoanal Study of the Child $24: 144-64,1969$.

8. Tolpin M: On the beginnings of a cohesive self. Psychoanal Study of the Child 26:316-52, 1971 . 